\title{
Association between Secondhand Smoke Exposure and Metabolic Syndrome in 118,609 Korean Never Smokers Verified by Self-Reported Questionnaire and Urine Cotinine
}

\author{
Ji Hye Kim¹, Byung Jin Kim², Young Youl Hyun, Jin Ho Kang² \\ Divisions of ${ }^{1}$ Nephrology, ${ }^{2}$ Cardiology, Department of Internal Medicine, Kangbuk Samsung Hospital, Sungkyunkwan \\ University School of Medicine, Seoul, Korea
}

Background: No study has reported the association between secondhand smoke (SHS) exposure and metabolic syndrome (MetS) in self-reported never smokers verified by both self-reported questionnaire and urine cotinine.

Methods: A total of 118,609 self-reported and cotinine-verified never smokers (38,385 male; age 34.8 \pm 7.1 years) who participated in the Kangbuk Samsung Health Study between 2011 and 2016 were included. Cotinine-verified never smokers were defined as individuals with urinary cotinine $<50 \mathrm{ng} / \mathrm{mL}$. SHS exposure was defined as current exposure to passive smoking indoors at home or workplace.

Results: Prevalence of SHS exposure in the overall population was $22.6 \%$ (27.4\% for males and $20.3 \%$ for females $(P<0.001)$. The overall prevalence of MetS was $6.8 \%$ and was higher in males than in females (10.7\% vs. $4.9 \%, P<0.001)$. In both genders, MetS prevalence was higher in the SHS exposure group than the non-SHS exposure group $(11.3 \%$ vs. $10.4 \%, P=0.010$ for males; $5.8 \%$ vs. $4.6 \%, P<0.001$ for females). However, there was significant gender interaction for the association between SHS exposure and MetS $(P$ for interaction $=0.010)$. In the multivariate regression analyses, SHS exposure was associated with increased MetS odds only in females (odds ratio [95\% confidence interval], 1.02 [0.94 to 1.11] in male vs. 1.17 [1.06 to 1.29] in female). In particular, females with SHS exposure of $\geq 1$ hour/day and $\geq 3$ times showed increased odds of MetS compared with those without SHS exposure (1.22 [1.02 to 1.45$], 1.30$ [1.14 to 1.49$])$.

Conclusion: This cross-sectional study showed that SHS exposure was significantly associated with prevalence of MetS in self-reported and cotinine-verified female never smokers.

Keywords: Tobacco products; Cotinine; Metabolic syndrome; Smoking; Surveys and questionnaires

\section{INTRODUCTION}

Cigarette smoking is a global, social, and medical hazard known as a strong risk factor for many cardiovascular and metabolic diseases including obesity, hypertension, dyslipidemia, and diabetes mellitus [1-4]. Of many toxic chemical constituents of
Received: 17 September 2020, Revised: 23 October 2020 ,

Accepted: 3 November 2020

Corresponding author: Byung Jin Kim

Division of Cardiology, Department of Internal Medicine, Kangbuk Samsung Hospital, Sungkyunkwan University School of Medicine, 29 Saemunan-ro, Jongno-gu, Seoul 03181, Korea

Tel: +82-2-2001-2001, Fax: +82-2-2001-2400, E-mail: bjjake.kim@samsung.com

\section{Copyright $(2020$ Korean Endocrine Society}

This is an Open Access article distributed under the terms of the Creative Commons Attribution Non-Commercial License (https://creativecommons.org/ licenses/by-nc/4.0/) which permits unrestricted non-commercial use, distribution, and reproduction in any medium, provided the original work is properly cited. 
cigarettes, nicotine is associated with high blood pressure, dyslipidemia, and insulin resistance through various pathophysiologic means including increased sympathetic tone, accumulation of abdominal fat and inflammation of pancreatic beta cells. All the above factors are the components of metabolic syndrome (MetS) [5-7]. Secondhand smoke (SHS) is also a public health problem accounting for 0.6 million deaths and approximately $1 \%$ of the global disease burden [8]. In Korea, even though SHS exposure prevalence has declined since 2005 due to various national health policies, overall indoor SHS exposure at the workplace and home in adults over 19 years of age is still $17.4 \%$. The indoor SHS prevalence is higher in males than in females (19.6\% in males vs. $14.8 \%$ in females) [9].

Accurate evaluation of actual smoking status is critical to assess the effects of cigarette smoking and SHS. However, several previous studies, which assessed impacts of cigarette smoking using standardized self-reported questionnaires, reported underestimation of actual smoking prevalence as some current or former smoking respondents accidentally or purposely responded to the questionnaires incorrectly as never smokers [10,11]. Cotinine is a biological metabolite of nicotine that can be measured in serum, saliva, or urine [12]. Urinary cotinine is a non-invasive biomarker with a half-life of 12 to 20 hours. Based on the presence of this biomarker in the urine, differentiation between never and current smokers can be achieved with a cut-off value of $50 \mathrm{ng} / \mathrm{mL}$ with high sensitivity and specificity [13-15].

As cigarette smoking is known to cause various metabolic dysfunctions associated with key components of MetS, we hypothesized that SHS exposure may also be associated with MetS [5-7]. To the best of our knowledge, there exists no study on the assessment of the association between SHS exposure in adults over 19 years of age and MetS in the Asian population. Therefore, this large cross-sectional study involving 118,609 Korean adults was conducted to evaluate the association between SHS exposure and MetS among self-reported and urinary cotinine-verified never smokers according to gender.

\section{METHODS}

\section{Study population}

Between 2011 and 2016, 134,231 self-reported never smokers from the Kangbuk Samsung Health Study (KSHS) were initially enrolled in the study. Among them, 2,497 were excluded due to presence of urinary cotinine over $50 \mathrm{ng} / \mathrm{mL}$, five were excluded due to missing urinary cotinine data and 13,130 were excluded due to missing waist circumference (WC) data. Finally,
118,609 self-reported and cotinine-verified never smokers with urinary cotinine $<50 \mathrm{ng} / \mathrm{mL}$ (38,385 male; age $34.8 \pm 7.1$ years) were enrolled in this study (Supplemental Fig. S1).

KSHS is an ongoing cohort study since 2002 including Koreans over 18 years of age, who have received comprehensive annual or biennial examinations at Total Healthcare Centers of Kangbuk Samsung Hospital. Written informed consent by the patients was waived due to a retrospective nature of our study. This study was approved by the Institutional Review Board of Kangbuk Samsung Hospital (IRB No: 2020-06-002).

\section{Anthropometry and laboratory tests}

A self-reported questionnaire was used to record underlying medical history including MetS, hypertension, and diabetes mellitus. Information on baseline smoking status (never, former, current smoking), status of current indoor SHS exposure at home or workplace, daily time of indoor SHS exposure (approximate hour and minute), weekly frequency of indoor SHS exposure (times per week), and total duration of indoor SHS exposure (approximate years and months). Information about the percentage of individuals who consumed alcohol more than 3 times per week and exercised more more than 5 times per week was also collected.

WC was measured at mid-level between the lowest rib and iliac crest. Body mass index (BMI) was calculated as weight (kilogram) divided by height (square meter). Systolic and diastolic blood pressures were measured by the hospital's registered nurse using a standardized sphygmomanometer.

Laboratory measurements including blood glucose, hemoglobin A1c (HbA1c), low-density lipoprotein cholesterol (LDL-C), high-density lipoprotein cholesterol (HDL-C), total cholesterol (TC), triglyceride (TG), high-sensitivity C-reactive protein (hsCRP), blood urea nitrogen (BUN), and uric acid were measured with an automated chemistry analyzer (Modular DPP, Roche Diagnostics, Tokyo, Japan) after at least 10 hours of the fasting period. Serum creatinine level was measured with Modular D2400 (Roche Diagnostics). Insulin resistance was defined using 'homeostatic model assessment of insulin resistance' $($ HOMA-IR $)=[$ fasting blood glucose $(\mathrm{mmol} / \mathrm{L}) \times$ fasting insulin $(\mu \mathrm{IU} / \mathrm{mL})] / 22.5$. Insulin secretory function was defined using 'homeostatic model assessment of $\beta$ cell function' $($ HOMA- $\beta)=$ [20 $\times$ fasting insulin $(\mu \mathrm{IU} / \mathrm{mL}) /$ fasting blood glucose $(\mathrm{mmol} /$ $\mathrm{mL})-3.5$ ]. Urinary cotinine was measured after at least 10 hours of the smoking free period using DRI Cotinine Assay (Microgenics, Fremont, CA, USA) and Modular P800 (Roche Diagnostics). 


\section{Definition of metabolic syndrome}

MetS was defined according to the joint interim statement issued in 2009 [16]. MetS was defined as having three or more of the following: (1) $\mathrm{WC}>90 \mathrm{~cm}$ for male and $>80 \mathrm{~cm}$ for female; (2) fasting TG concentrations $\geq 150 \mathrm{mg} / \mathrm{dL}$ or treatment with TG lowering medications; (3) HDL-C concentrations $<40$ $\mathrm{mg} / \mathrm{dL}$ for male and $<50 \mathrm{mg} / \mathrm{dL}$ for female; (4) systolic blood pressure $\geq 130 \mathrm{~mm} \mathrm{Hg}$ and/or diastolic blood pressure $\geq 85 \mathrm{~mm}$ $\mathrm{Hg}$ or taking antihypertensive medication; and (5) fasting glucose levels $\geq 100 \mathrm{mg} / \mathrm{dL}$ or taking antidiabetic medication.

\section{Definition of smoking status and SHS exposure}

According to the self-reported questionnaire, self-reported never smoker was defined as never smoked or had smoked less than a total of five cigarette packs in one's life. Cotinine-verified never smoker was defined as having urinary cotinine $<50 \mathrm{ng} / \mathrm{mL}$ after 10 hours of smoking free period. This cut-off point was used to classify current smokers from never smokers according to the recommendations from Society for Research on Nicotine and Tobacco (SRNT) [15]. Our previous studies show that this cutoff level has high sensitivity $(84.8 \%)$ and specificity $(98.2 \%)$ in differentiating never from current smokers [13]. SHS exposure was defined as currently experiencing passive smoking at home or the workplace.

\section{Statistical analysis}

Continuous variables are expressed as mean \pm standard deviation or as median with interquartile ranges. Categorical variables are expressed as percentages (\%). Serum TG, hsCRP, and HOMA-IR were log-transformed to correct for skewed distributions. Data in tables are expressed as untransformed raw data for easier interpretation.

Comparative analyses of baseline characteristics between the groups according to SHS exposure and MetS status were conducted using Student's $t$ test or chi-square test. Multivariate logistic regression analyses were performed to assess the association between SHS exposure and MetS and MetS components (WC, HDL-C, fasting TG, fasting glucose, systolic and diastolic blood pressure). Also, the associations between SHS exposure and MetS according to daily time, weekly freqeuncy, and total duration of SHS exposure were assessed. The multivariate model was adjusted for variables with a univariate relationship $(P<0.05)$, including age, BMI, frequency of alcohol drinking, frequency of vigorous exercise, BUN, creatinine, uric acid, and hsCRP. Statistical significance was valid when the $P$ value was less than 0.05 . The above statistical analyses were performed with IBM SPPS version 24 (IBM Corp., Armonk, NY, USA).

\section{RESULTS}

\section{Prevalence of SHS exposure and baseline characteristics} according to SHS exposure status

The overall percentage of individuals with SHS exposure was $22.6 \%$ (Supplemental Table S1). SHS exposure prevalence was higher in males than in females $(27.4 \%$ in males and $20.3 \%$ in females) (Supplemental Fig. S2). However, among individuals with SHS exposure, percentage of individuals with SHS exposure for more than 1 hour/day (26.7\% vs. 37.9\%), 3 times/week (32.4\% vs. $37.9 \%$ ), and 10 years (55.6\% vs. 63.4\%) was higher in females (Table 1).

In males, SHS exposure group were slightly younger with higher BMI, WC, systolic and diastolic blood pressure, glucose, HbA1c, and lower LDL-C, and HOMA- $\beta$ levels. In females, SHS exposure group were slightly younger with higher BMI, WC, systolic and diastolic blood pressure, HDL-C, glucose, $\mathrm{HbA1c}$, and lower LDL-C levels. Percentage of individuals who exercised and drank alcohol more than 3 times/week and had hypertension, diabetes mellitus, and MetS was higher in both male and female SHS exposure groups (Table 1).

\section{Prevalence of MetS and baseline characteristics according to MetS status}

The overall MetS prevalence was 6.8\%. MetS prevalence in males was approximately two times higher $(10.7 \%$ in male vs $4.9 \%$ in female) (Supplemental Fig. S3). In males, those with MetS were older with higher BMI, WC, systolic and diastolic blood pressure, TC, TG, LDL-C, glucose, HbAlc, HOMA-IR, and lower HDL-C and HOMA- $\beta$, which are the major components of MetS diagnosis (Supplemental Table S2). Similar findings were seen throughout the comparison of variables in females. Females with MetS were also older with higher BMI, WC, systolic and diastolic blood pressure, TC, TG, HDL-C, LDL-C, glucose, HbA1c, HOMA-IR, and lower HOMA- $\beta$ level (Supplemental Table S2).

\section{Association between SHS exposure and MetS \& MetS components according to gender}

In the overall study population, there was significant association between SHS exposure and MetS in both age- and sex-adjusted (odds ratio [OR], 1.19; 95\% confidence interval [CI], 1.13 to $1.25)$ and multivariate $(\mathrm{OR}, 1.09 ; 95 \% \mathrm{CI}, 1.02$ to 1.16$)$ regression analyses (Supplemental Table S3). However, because there 
Table 1. Characteristics of Male and Female Never Smokers with and without Secondhand Smoke Exposure

\begin{tabular}{|c|c|c|c|c|c|c|}
\hline \multirow[b]{2}{*}{ Characteristic } & \multicolumn{3}{|c|}{ Male never smokers } & \multicolumn{3}{|c|}{ Female never smokers } \\
\hline & $\begin{array}{c}\text { SHS exposure }(-) \\
\quad(n=27,854)\end{array}$ & $\begin{array}{c}\text { SHS exposure }(+) \\
\quad(n=10,531)\end{array}$ & $P$ value & $\begin{array}{c}\text { SHS exposure }(-) \\
\quad(n=63,917)\end{array}$ & $\begin{array}{c}\text { SHS exposure }(+) \\
\quad(n=16,307)\end{array}$ & $P$ value \\
\hline Age, yr & $34.4 \pm 6.4$ & $34.3 \pm 6.2$ & 0.152 & $35.1 \pm 7.3$ & $34.7 \pm 8.0$ & $<0.001$ \\
\hline Body mass index, $\mathrm{kg} / \mathrm{m}^{2}$ & $24.3 \pm 3.0$ & $24.4 \pm 3.0$ & $<0.001$ & $21.5 \pm 3.0$ & $21.7 \pm 3.3$ & $<0.001$ \\
\hline Waist circumference, $\mathrm{cm}$ & $85.1 \pm 8.2$ & $85.6 \pm 8.2$ & $<0.001$ & $75.0 \pm 8.1$ & $75.7 \pm 8.7$ & $<0.001$ \\
\hline Systolic blood pressure, $\mathrm{mm} \mathrm{Hg}$ & $113.6 \pm 10.9$ & $114.2 \pm 10.8$ & $<0.001$ & $100.7 \pm 10.5$ & $101.1 \pm 10.7$ & $<0.001$ \\
\hline Diastolic blood pressure, $\mathrm{mm} \mathrm{Hg}$ & $72.1 \pm 8.9$ & $72.5 \pm 9.1$ & $<0.001$ & $64.3 \pm 8.0$ & $64.7 \pm 8.3$ & $<0.001$ \\
\hline Blood urea nitrogen, mmol/L & $4.53 \pm 1.05$ & $4.58 \pm 1.05$ & $<0.001$ & $4.06 \pm 1.06$ & $4.06 \pm 1.05$ & 0.874 \\
\hline Serum creatinine, $\mu \mathrm{mol} / \mathrm{L}$ & $86.5 \pm 11.1$ & $87.1 \pm 11.1$ & $<0.001$ & $60.4 \pm 11.3$ & $61.3 \pm 10.2$ & $<0.001$ \\
\hline Uric acid, $\mu \mathrm{mol} / \mathrm{L}$ & $370.5 \pm 72.7$ & $371.3 \pm 73.4$ & 0.363 & $252.9 \pm 53.3$ & $255.5 \pm 53.9$ & $<0.001$ \\
\hline Total cholesterol, $\mathrm{mmol} / \mathrm{L}$ & $5.04 \pm 0.86$ & $5.04 \pm 0.86$ & 0.499 & $4.76 \pm 0.81$ & $4.76 \pm 0.81$ & 0.691 \\
\hline Triglyceride, $\mathrm{mmol} / \mathrm{L}$ & $1.08(0.79-1.55)$ & $1.08(0.79-1.56)$ & 0.296 & $0.77(0.60-1.03)$ & $0.76(0.59-1.04)$ & 0.333 \\
\hline $\mathrm{HDL}-\mathrm{C}, \mathrm{mmol} / \mathrm{L}$ & $1.40 \pm 0.33$ & $1.41 \pm 0.33$ & 0.705 & $1.71 \pm 0.38$ & $1.71 \pm 0.39$ & 0.017 \\
\hline LDL-C, mmol/L & $3.27 \pm 0.80$ & $3.25 \pm 0.80$ & 0.023 & $2.80 \pm 0.74$ & $2.78 \pm 0.75$ & 0.008 \\
\hline Glucose, $\mathrm{mmol} / \mathrm{L}$ & $5.24 \pm 0.67$ & $5.26 \pm 0.73$ & 0.003 & $5.02 \pm 0.56$ & $5.04 \pm 0.64$ & $<0.001$ \\
\hline Hemoglobin A1c, \% (mmol/mol) & $5.52 \pm 0.42$ & $5.54 \pm 0.43$ & 0.023 & $5.52 \pm 0.35$ & $5.54 \pm 0.39$ & $<0.001$ \\
\hline HOMA-IR & $1.28(0.85-1.90)$ & $1.26(0.83-1.89)$ & 0.059 & $1.11(0.74-1.61)$ & $1.10(0.73-1.64)$ & 0.878 \\
\hline HOMA- $\beta$ & $11.7(10.0-13.8)$ & $11.5(9.9-13.7)$ & 0.003 & $13.2(11.2-16.1)$ & $13.2(11.1-16.0)$ & $<0.001$ \\
\hline hsCRP, mg/L & $0.5(0.3-1.0)$ & $0.5(0.3-1.0)$ & 0.019 & $0.3(0.2-0.7)$ & $0.3(0.2-0.7)$ & 0.668 \\
\hline Vigorous exercise & & & $<0.001$ & & & $<0.001$ \\
\hline None & $14,326 / 27,635(51.8)$ & $5,104 / 10,464(48.8)$ & & $46,480 / 63,032(73.7)$ & $10,852 / 16,054(67.6)$ & \\
\hline$<3$ times/wk & $9,442 / 27,635(34.2)$ & $3,842 / 10,464(36.7)$ & & $10235 / 63,032(16.2)$ & $3,297 / 16,054(20.5)$ & \\
\hline$\geq 3$ times/wk & $3,867 / 27,635(14.0)$ & $1,518 / 10,464(14.5)$ & & $6,317 / 63,032(10.0)$ & 1,905/16,054 (11.9) & \\
\hline Alcohol consumption & & & $<0.001$ & & & $<0.001$ \\
\hline None & $1,961 / 27,539(7.1)$ & $546 / 10,391(5.3)$ & & $12,325 / 60,145(20.5)$ & $2,335 / 15,479(15.1)$ & \\
\hline $1-2$ times/wk & $23,021 / 27,539(83.6)$ & 8,273/10,391 (79.6) & & $44,598 / 60,145(74.2)$ & $11,730 / 15,479(75.8)$ & \\
\hline 3-4 times/wk & $2,265 / 27,539(8.2)$ & 1,413/10,391 (13.6) & & $2,697 / 60,145(4.5)$ & $1,187 / 15,479(7.7)$ & \\
\hline$\geq 5$ times $/ \mathrm{wk}$ & $292 / 27,539(1.1)$ & $159 / 10,391(1.5)$ & & $525 / 60,145(0.9)$ & $227 / 15,479(1.5)$ & \\
\hline Daily times of SHS exposure & & & $<0.001$ & & & $<0.001$ \\
\hline None & $27,854 / 27,854(100)$ & $0 / 5,526(0)$ & & 63,917/63,917 (100) & $0 / 8,841(0)$ & \\
\hline$<1 \mathrm{hr} /$ day & $0 / 27,854(0)$ & $4,051 / 5,526(73.2)$ & & 0/63,917 (0) & $5,489 / 8,841(62.1)$ & \\
\hline$\geq 1 \mathrm{hr} /$ day & $0 / 27,854(0)$ & $1,475 / 5,526(26.7)$ & & 0/63,917 (0) & $3,352 / 8,841(37.9)$ & \\
\hline Frequency of SHS exposure & & & $<0.001$ & & & $<0.001$ \\
\hline None & 27,814/27,814 (100) & $0 / 10,460(0)$ & & $63,858 / 63,858(100)$ & $0 / 16,076(0)$ & \\
\hline$<3$ times/wk & $0 / 27,814(0)$ & $7,071 / 10,460(67.6)$ & & $0 / 63,858(0)$ & $10,300 / 16,076(64.1)$ & \\
\hline$\geq 3$ times/wk & $0 / 27,814(0)$ & $3,389 / 10,460(32.4)$ & & $0 / 63,858(0)$ & $5,776 / 16,076(35.9)$ & \\
\hline Duration of SHS exposure & & & $<0.001$ & & & $<0.001$ \\
\hline None & $27,766 / 27,766(100)$ & $0 / 7,048(0)$ & & $63,507 / 63,507(100)$ & $0 / 10,853(0)$ & \\
\hline$<10 \mathrm{yr}$ & $0 / 27,766(0)$ & $3,132 / 7,048(44.4)$ & & $0 / 63,507(0)$ & $3,970 / 10,853(36.6)$ & \\
\hline$\geq 10 \mathrm{yr}$ & $0 / 27,766(0)$ & $3,916 / 7,048(55.6)$ & & $0 / 63,507(0)$ & $6,883 / 10,853(63.4)$ & \\
\hline Hypertension & 2,893/27,854 (10.4) & 1,252/10,531 (11.9) & $<0.001$ & 2,064/63,917 (3.2) & 638/16,307 (3.9) & $<0.001$ \\
\hline Diabetes mellitus & $577 / 27,852(2.1)$ & $223 / 10,528(2.1)$ & 0.776 & $752 / 63,911(1.2)$ & $284 / 16,305(1.7)$ & $<0.001$ \\
\hline Metabolic syndrome & 2,903/27,854 (10.4) & 1,194/10,531 (11.3) & 0.010 & 2,971/63,917 (4.6) & $943 / 16,307(5.8)$ & $<0.001$ \\
\hline
\end{tabular}

Values are expressed as mean \pm standard deviation, median (interquartile range), or number (\%). Triglyceride, HOMA-IR, HOMA- $\beta$, and hsCRP and daily alcohol amount were log-transformed for this analysis. $P$ values are based on Student's $t$ test or chi-square test.

SHS, secondhand smoke; HDL-C, high-density lipoprotein cholesterol; LDL-C, low-density lipoprotein cholesterol; HOMA-IR, homeostatic model assessment of insulin resistance; HOMA- $\beta$, homeostatic model assessment of $\beta$ cell function; hsCRP, high-sensitivity C-reactive protein. 
Table 2. Multivariate Logistic Regression Analyses for the Association of Metabolic Syndrome and Its Components with Secondhand Smoke Exposure in Male and Female Never Smokers

\begin{tabular}{lccccc}
\hline \multirow{2}{*}{ Variable } & \multicolumn{2}{c}{ Male never smokers } & & \multicolumn{2}{c}{ Female never smokers } \\
\cline { 2 - 3 } \cline { 5 - 6 } Metabolic syndrome & Age-adjusted & Multivariate & & Age-adjusted & Multivariate \\
Metabolic syndrome components & $1.11(1.04-1.20)$ & $1.02(0.94-1.11)$ & & $1.27(1.17-1.37)$ & $1.17(1.06-1.29)$ \\
Fasting glucose $\geq 100 \mathrm{mg} / \mathrm{dL}$ & $1.10(1.04-1.16)$ & $1.04(0.98-1.10)$ & & $1.14(1.08-1.21)$ & $1.09(1.02-1.16)$ \\
WC $>90 \mathrm{~cm}$ & $1.11(1.06-1.17)$ & $1.02(0.94-1.11)$ & & $1.22(1.18-1.27)$ & $1.12(1.05-1.19)$ \\
HDL-C $<40 \mathrm{mg} / \mathrm{dL}$ & $1.02(0.95-1.10)$ & $1.03(0.96-1.12)$ & & $1.09(1.04-1.15)$ & $1.07(1.01-1.13)$ \\
Fasting TG $\geq 150 \mathrm{mg} / \mathrm{dL}$ & $1.04(0.99-1.10)$ & $1.01(0.95-1.07)$ & & $1.14(1.06-1.22)$ & $1.06(1.01-1.15)$ \\
SBP $\geq 130 \mathrm{and} / \mathrm{or} \mathrm{DBP} \geq 85 \mathrm{~mm} \mathrm{Hg}$ & $1.07(1.01-1.15)$ & $1.01(0.94-1.08)$ & & $1.19(1.09-1.30)$ & $1.23(1.02-1.24)$ \\
\hline
\end{tabular}

Values are expressed as odds ratio (95\% confidence interval). Multivariate model was adjusted for age, body mass index, frequency of alcohol drinking, frequency of vigorous exercise, blood urea nitrogen, creatinine, uric acid, total cholesterol, low-density lipoprotein cholesterol, and high-sensitivity C-reactive protein.

WC, waist circumference; HDL-C, high-density lipoprotein cholesterol; TG, triglyceride; SBP, systolic blood pressure; DBP, diastolic blood pressure.

was a significant gender interaction in SHS exposure and MetS ( $P$ for interaction $<0.001$ ), the analyses were conducted separately according to gender.

In the age-adjusted analysis, there were significant associations between SHS exposure and MetS in both genders with higher odds in females (OR, 1.11 [95\% CI, 1.04 to 1.20] in males vs. OR, 1.27 [95\% CI, 1.17 to 1.37 in females). However, multivariate analyses revealed a significant association between SHS expousre and increased MetS only in females (OR, 1.02 [95\% CI, 0.94 to 1.11] in males vs. OR, 1.17 [95\% CI, $1.06,1.29]$ in females) (Table 2).

In the age-adjusted regression analyses for the five components of MetS, males with SHS exposure had increased odds for high fasting glucose (fasting glucose $\geq 100 \mathrm{mg} / \mathrm{dL}$; OR, 1.10; $95 \% \mathrm{CI}, 1.04$ to 1.16 ), abdominal obesity (WC $>90 \mathrm{~cm}$; OR, $1.11 ; 95 \% \mathrm{CI}, 1.06$ to 1.17 ) and hypertension (SBP $\geq 130 \mathrm{~mm}$ $\mathrm{Hg}$ and/or DBP $\geq 85 \mathrm{~mm} \mathrm{Hg}$; OR, 1.07; 95\% CI, 1.01 to 1.15 ) compared to the non-SHS exposure group. However, in the multivariate analyses of MetS components, males with SHS exposure had no statistically significant odds compared to the nonSHS exposure group (Table 2).

In contrast, females with SHS exposure had increased odds for all five components of MetS compared to the non-SHS exposure group in both age-adjusted and multivariate analyses (Table 2).

\section{Association between SHS exposure and MetS according to daily time, frequency, and duration of SHS exposure}

In males, trend for increasing MetS odds according to increas- ing daily hour, weekly frequency, and total duration of SHS exposure was observed in the age-adjusted model ( $P$ for trend all $<0.005$ ), but was not statistically significant in the multivariate model (Table 3).

In females, trend for increasing MetS odds according to increasing daily hour and weekly frequency was seen in both ageadjusted and multivariate models ( $P$ for trend all $<0.005$ ). In particular, females who were exposed for more than $\geq 1$ hour/ day and $\geq 3$ times/week increased MetS odds by $22 \%$ and $30 \%$, respectively (OR, 1.22 [95\% CI, 1.02 to 1.45]; OR, 1.30 [95\% CI, 1.14 to 1.49$]$ ) (Table 3).

\section{DISCUSSION}

The results of this study showed that SHS exposure in female self-reported and cotinine-verified never smokers was significantly associated with increased MetS. This association was dose-dependent on increasing daily hour and weekly frequency of SHS exposure in females. In particular, female never smokers with SHS exposure for more than 1 hour/day and 3 times/ week had a $20 \%$ to $30 \%$ higher risk of MetS compared to nonSHS exposure females.

MetS is a group of metabolic abnormalities including central obesity, insulin resistance, dyslipidemia and hypertension. It is associated with increased risks of type 2 diabetes mellitus and various cardiovascular diseases [17]. The pathophysiologic mechanism of MetS development is not yet fully understood. However, central obesity and insulin resistance are known as main causative triggers along with various socioeconomic, ge- 
Table 3. Multivariate Logistic Regression Analyses for the Association between Metabolic Syndrome and Secondhand Smoke Exposure According to Daily Time, Frequency, and Duration in Male and Female Never Smokers

\begin{tabular}{|c|c|c|c|c|}
\hline \multirow{2}{*}{ Variable } & \multicolumn{2}{|c|}{ Male never smokers } & \multicolumn{2}{|c|}{ Female never smokers } \\
\hline & Age-adjusted & Multivariate & Age-adjusted & Multivariate \\
\hline \multicolumn{5}{|c|}{ Daily time of SHS exposure } \\
\hline None & 1 (Reference) & 1 (Reference) & 1 (Reference) & 1 (Reference) \\
\hline$<1 \mathrm{hr}$ & $0.96(0.85-1.07)$ & $0.83(0.73-0.95)$ & $1.28(1.11-1.47)$ & $1.03(0.87-1.22)$ \\
\hline$\geq 1 \mathrm{hr}$ & $1.48(1.28-1.72)$ & $1.18(0.99-1.14)$ & $1.48(1.29-1.71)$ & $1.22(1.02-1.45)$ \\
\hline$P$ for trend & $<0.001$ & 0.961 & $<0.001$ & 0.041 \\
\hline \multicolumn{5}{|c|}{ Frequency of SHS exposure } \\
\hline None & 1 (Reference) & 1 (Reference) & 1 (Reference) & 1 (Reference) \\
\hline$<3$ times/wk & $1.03(0.94-1.12)$ & $1.00(0.91-1.11)$ & $1.11(1.00-1.23)$ & $1.07(0.94-1.21)$ \\
\hline$\geq 3$ times/wk & $1.29(1.16-1.44)$ & $1.07(0.94-1.21)$ & $1.51(1.35-1.68)$ & $1.30(1.14-1.49)$ \\
\hline$P$ for trend & $<0.001$ & 0.414 & $<0.001$ & $<0.001$ \\
\hline \multicolumn{5}{|c|}{ Duration of SHS exposure } \\
\hline None & 1 (Reference) & 1 (Reference) & 1 (Reference) & 1 (Reference) \\
\hline$<10 \mathrm{yr}$ & $1.03(0.91-1.17)$ & $0.94(0.81-1.09)$ & $1.22(1.03-1.45)$ & $1.13(0.93-1.38)$ \\
\hline$\geq 10 \mathrm{yr}$ & $1.18(1.06-1.31)$ & $0.97(0.86-1.10)$ & $1.32(1.19-1.47)$ & $1.12(0.99-1.28)$ \\
\hline$P$ for trend & 0.004 & 0.981 & $<0.001$ & 0.051 \\
\hline
\end{tabular}

netic and environmental factors [18,19]. The kew environmental factors associated with MetS are active smoking and SHS exposure $[1,5,20,21]$.

Cigarette smoke is an important environmental trigger factor associated with activation and aggravation of key components of MetS. In exploration of the association between smoking and each key component of MetS, it has been identified that smoking is associated with central obesity by dose-dependent abdominal fat accumulation through increased cortisol levels, insulin resistance and decreased estrogen levels thereby leading to hormonal imbalance in both the genders [22-25]. Smoking increases serum glucose levels mainly through chronic inflammation and direct toxic dysfunction of pancreatic beta cells $[2,26,27]$. It also promotes dyslipidemia by increasing oxidative stress and decreasing level of antioxidants resulting in higher TC, TG, LDL-C, and lower HDL-C levels [28-30].

Multiple studies which assessed the association between smoking and hypertension have shown inconsistent results [4,13,31-33]. However, a recent cross-sectional study which assessed the association between SHS exposure and hypertension in Korean adults has shown that SHS exposure is associated with increased odds for hypertension [34]. The pathophysiology behind this association is mainly nicotine-mediated sympathetic activation, endothelial dysfunction, hypercoagulation, and increased arterial stiffness [31,34,35].

The pathophysiology behind increased MetS prevalence in never smokers with SHS exposure is suspected to be similar to that of active smoking. Many previous studies have evaluated the association between smoking and MetS and demonstrated a positive association between the two [1,5,20,30]. Our study also showed a positive association between SHS exposure and MetS in females. To our knowledge, this is the first study that assessed the association between SHS exposure and MetS in Asian adults using a dual smoking status verification system to increase the accuracy of actual smoking status classification.

An interesting finding in our study is that the association between SHS and MetS was only significant in females (OR, 1.02 [95\% CI, 0.94 to 1.11] in males vs. OR, 1.17 [95\% CI, 1.06 to 1.29] in females). C-statistic value of SHS for MetS was also higher in females (OR, 0.86 [95\% CI, 0.86 to 0.87] for males vs. OR, 0.92 [95\% CI, 0.92 to 0.93 ] for females). This finding is in correlation with the result that female SHS exposed never 
smokers had increased odds for all the five components of MetS compared to female non-SHS exposed group, while the association was not valid in all five components in male never smokers (Table 2). It is hypothesized that this finding was due to the that fact that even though percentage of those with SHS exposure was higher in males, percentage of female never smokers with SHS exposure who were exposed for more than 1 hour/day, 3 times/week, and total 10 years was higher. Cotinine metabolism rate is higher in females than males due to higher estrogen level $[12,36]$. Therefore, even though both male and female self-reported and cotinine verified never smokers had urine cotinine under $50 \mathrm{ng} / \mathrm{mL}$, female SHS exposed never smokers may have had higher amount, frequency and duration of SHS exposure than male SHS exposed never smokers as shown in our study results. Also, the non-SHS exposed male never smokers had more unfavorable baseline characteristics inclined towards MetS including higher BMI, WC, SBP/DBP, glucose, and abnormal lipid profile. Lastly, the percentage of individuals who consumed alcohol for more than 3 times/week was nearly twice higher in male never smokers compared to female never smokers in both SHS exposure and non-SHS exposure groups. As moderate alcohol consumption is known to be associated with decreased MetS prevalence this might have served as a protective confounding factor for MetS [37,38].

Previous studies have suspected that key mechanisms through which cigarette smoking acts on MetS development are central obesity and insulin resistance [18-21]. Abdominal obesity, which is represented by WC, was a statistically valid MetS component with second-highest odds among the five MetS components in female SHS exposed never smokers. The results of this study also correlate with the results from the above studies that SHS exposure has a strong association with central obesity and this may be one of the key factors mediating the association between SHS exposure and MetS.

This study has some limitations. First, MetS prevalence in this study was relatively lower than that in Korean adults. According to the 'Metabolic syndrome fact sheet Korea 2018' which analyzed data from the 2007 to 2015 Korea National Health and Nutritional Examination Survey, overall MetS prevalence in 2015 in Koreans aged over 19 years of age was 22.4\% [39]. However, MetS prevalence in this study was $6.8 \%$. Relatively underrated MetS prevalence in this study may be because MetS prevalence tends to increase with age usually peaking at age 50 to 60 years while the average age of this study's population was mid-thirties in both genders. Unfortunately, only socioeconomic information about monthly income and educational levels were available. In the multivariate analyses including the above two socioeconomic status variables, similar trend of increased odds of MetS in female never smokers with SHS exposure was observed (OR, 1.03 [95\% CI, 0.93 to 1.14] for male and OR, 1.19 [95\% CI, 1.05, 1.35] for female; data not shown). However, $30 \%$ of the study cohort did not provide information regarding socioeconomic status, which could act as statistical and selection bias in the analyses and interpretation of this study. Therefore, we did not include the above variables to analyse the main results of our study, but the importance of collecting data including socioeconomic status and dietary patterns is noted.

Second, it is a cross-sectional study. Therefore, no causal association between SHS exposure and MetS can be concluded from this study. Third, the metabolism of urine cotinine is affected by various racial, genetic, and gender factors. For example, females and especially pregnant females have much higher nicotine metabolism rate compared to males $[12,15,36,40]$. As the mean age of females in this study was mid-30s there is a possibility that a certain portion of the female population were pregnant or had higher nicotine metabolism rate due to gender difference. Fourth, no information on underlying diseases other than diabetes mellitus, hypertension and MetS was collected. Endocrinologic diseases such as polycystic ovary syndrome (PCOS) are known to be independently associated with MetS $[41,42]$. PCOS is common in females of reproductive age and as the mean age of this study population was mid-thirties this could have acted as a confounding factor. Nonetheless this study has its strength as it is the first large cross-sectional study that accurately analyzed the association between SHS exposure and MetS in Korean adults using two different smoking verification systems; self-reported questionnaire and urinary cotinine level.

In conclusion, this large cross-sectional study revealed that SHS was significantly associated with MetS in a dose-dependent manner in self-reported and urinary cotinine verified female never smokers. More active regional and national campaigns should be implemented to build awareness about smoking and prevent SHS at both home and workplace to reduce MetS. Further longitudinal studies using the above two smoking verification systems are needed to clarify the causal association between SHS exposure and MetS.

\section{CONFLICTS OF INTEREST}

No potential conflict of interest relevant to this article was reported. 


\section{ACKNOWLEDGMENTS}

This study was published in abstract form in 2019 ESC Congress and presented in 'Poster' section of the congress.

\section{AUTHOR CONTRIBUTIONS}

Conception or design: J.H.K., B.J.K. Acquisition, analysis, or interpretation of data: J.H.K., B.J.K., Y.Y.H., J.H.K. Drafting the work or revising: J.H.K., B.J.K., Y.Y.H., J.H.K. Final approval of the manuscript: J.H.K., B.J.K., Y.Y.H., J.H.K.

\section{ORCID}

Ji Hye Kim https://orcid.org/0000-0002-3912-7709

Byung Jin Kim https://orcid.org/0000-0002-9008-8506

\section{REFERENCES}

1. Oh SW, Yoon YS, Lee ES, Kim WK, Park C, Lee S, et al. Association between cigarette smoking and metabolic syndrome: the Korea National Health and Nutrition Examination Survey. Diabetes Care 2005;28:2064-6.

2. Kim JH, Kim BJ, Kang JG, Kim BS, Kang JH. Association between cigarette smoking and diabetes mellitus using two different smoking stratifications in 145040 Korean individuals: self-reported questionnaire and urine cotinine concentrations. J Diabetes 2019;11:232-41.

3. Shin DY, Jang YK, Lee JH, Wee JH, Chun DH. Relationship with smoking and dyslipidemia in Korean adults. J Korean Soc Res Nicotine Tob 2017;8:73-9.

4. Virdis A, Giannarelli C, Neves MF, Taddei S, Ghiadoni L. Cigarette smoking and hypertension. Curr Pharm Des 2010; 16:2518-25.

5. Kim BJ, Kang JG, Han JM, Kim JH, Lee SJ, Seo DC, et al. Association of self-reported and cotinine-verified smoking status with incidence of metabolic syndrome in 47379 Korean adults. J Diabetes 2019;11:402-9.

6. Madika AL, Mounier-Vehier C. Smoking and blood pressure: a complex relationship. Presse Med 2017;46(7-8 Pt 1): 697-702.

7. Li Z, Xu W, Su Y, Gao K, Chen Y, Ma L, et al. Nicotine induces insulin resistance via downregulation of Nrf2 in cardiomyocyte. Mol Cell Endocrinol 2019;495:110507.

8. World Health Organization. Secondhand smoke [Internet]. Geneva: WHO; 2020 [cited 2020 Nov 17]. Available from: https://www.who.int/gho/phe/secondhand_smoke/en/.

9. Ministry of Health \& Welfare. Korea Health Statistics 2016: Korea National Health and Nutrition Examination Survey (KNHANES VII-1). Sejong: Ministry of Health \& Welfare; 2017.

10. Hwang JH, Kim JY, Lee DH, Jung HG, Park SW. Underestimation of self-reported smoking prevalence in Korean adolescents: evidence from gold standard by combined method. Int J Environ Res Public Health 2018;15:689.

11. Connor Gorber S, Schofield-Hurwitz S, Hardt J, Levasseur G, Tremblay M. The accuracy of self-reported smoking: a systematic review of the relationship between self-reported and cotinine-assessed smoking status. Nicotine Tob Res 2009;11:12-24.

12. Benowitz NL, St Helen G, Nardone N, Cox LS, Jacob P. Urine metabolites for estimating daily intake of nicotine from cigarette smoking. Nicotine Tob Res 2020;22:288-92.

13. Kim BJ, Seo DC, Kim BS, Kang JH. Relationship between cotinine-verified smoking status and incidence of hypertension in 74,743 Korean adults. Circ J 2018;82:1659-65.

14. Parker DR, Lasater TM, Windsor R, Wilkins J, Upegui DI, Heimdal J. The accuracy of self-reported smoking status assessed by cotinine test strips. Nicotine Tob Res 2002;4:305-9.

15. SRNT Subcommittee on Biochemical Verification. Biochemical verification of tobacco use and cessation. Nicotine Tob Res 2002;4:149-59.

16. Alberti KG, Eckel RH, Grundy SM, Zimmet PZ, Cleeman JI, Donato KA, et al. Harmonizing the metabolic syndrome: a joint interim statement of the International Diabetes Federation Task Force on Epidemiology and Prevention; National Heart, Lung, and Blood Institute; American Heart Association; World Heart Federation; International Atherosclerosis Society; and International Association for the Study of Obesity. Circulation 2009;120:1640-5.

17. Rochlani Y, Pothineni NV, Kovelamudi S, Mehta JL. Metabolic syndrome: pathophysiology, management, and modulation by natural compounds. Ther Adv Cardiovasc Dis 2017;11:215-25.

18. Matsuzawa Y, Funahashi T, Nakamura T. The concept of metabolic syndrome: contribution of visceral fat accumulation and its molecular mechanism. J Atheroscler Thromb 2011;18:629-39.

19. O’Neill S, O’Driscoll L. Metabolic syndrome: a closer look at the growing epidemic and its associated pathologies. Obes Rev 2015;16:1-12.

20. Sun K, Liu J, Ning G. Active smoking and risk of metabolic 
syndrome: a meta-analysis of prospective studies. PLoS One 2012;7:e47791.

21. Weitzman M, Cook S, Auinger P, Florin TA, Daniels S, Nguyen $\mathrm{M}$, et al. Tobacco smoke exposure is associated with the metabolic syndrome in adolescents. Circulation 2005;112:862-9.

22. Kim Y, Jeong SM, Yoo B, Oh B, Kang HC. Associations of smoking with overall obesity, and central obesity: a crosssectional study from the Korea National Health and Nutrition Examination Survey (2010-2013). Epidemiol Health 2016;38:e2016020.

23. Tanko LB, Christiansen C. An update on the antiestrogenic effect of smoking: a literature review with implications for researchers and practitioners. Menopause 2004;11:104-9.

24. Lv J, Chen W, Sun D, Li S, Millwood IY, Smith M, et al. Gender-specific association between tobacco smoking and central obesity among 0.5 million Chinese people: the China Kadoorie Biobank Study. PLoS One 2015;10:e0124586.

25. Clair C, Chiolero A, Faeh D, Cornuz J, Marques-Vidal P, Paccaud F, et al. Dose-dependent positive association between cigarette smoking, abdominal obesity and body fat: cross-sectional data from a population-based survey. BMC Public Health 2011;11:23.

26. Chang SA. Smoking and type 2 diabetes mellitus. Diabetes Metab J 2012;36:399-403.

27. Akter S, Goto A, Mizoue T. Smoking and the risk of type 2 diabetes in Japan: a systematic review and meta-analysis. J Epidemiol 2017;27:553-61.

28. Craig WY, Palomaki GE, Haddow JE. Cigarette smoking and serum lipid and lipoprotein concentrations: an analysis of published data. BMJ 1989;298:784-8.

29. Yan-Ling Z, Dong-Qing Z, Chang-Quan H, Bi-Rong D. Cigarette smoking and its association with serum lipid/lipoprotein among Chinese nonagenarians/centenarians. Lipids Health Dis 2012;11:94.

30. Kolovou GD, Kolovou V, Mavrogeni S. Cigarette smoking/ cessation and metabolic syndrome. Clin Lipidol 2016;11:614.

31. Rhee MY, Na SH, Kim YK, Lee MM, Kim HY. Acute effects of cigarette smoking on arterial stiffness and blood pressure in male smokers with hypertension. Am J Hyper- tens 2007;20:637-41.

32. Kim BJ, Han JM, Kang JG, Kim BS, Kang JH. Association between cotinine-verified smoking status and hypertension in 167,868 Korean adults. Blood Press 2017;26:303-10.

33. Dong-Qing Z, Chang-Quan H, Yan-Ling Z, Bi-Rong D, Qing-Xiu L. Cigarette smoking is associated with increased diastolic blood pressure among Chinese nonagenarians/centenarians. Blood Press 2014;23:168-73.

34. Kim BJ, Kang JG, Kim JH, Seo DC, Sung KC, Kim BS, et al. Association between secondhand smoke exposure and hypertension in 106,268 Korean self-reported never-smokers verified by cotinine. J Clin Med 2019;8:1238.

35. Groppelli A, Giorgi DM, Omboni S, Parati G, Mancia G. Persistent blood pressure increase induced by heavy smoking. J Hypertens 1992;10:495-9.

36. Benowitz NL, Hukkanen J, Jacob P 3rd. Nicotine chemistry, metabolism, kinetics and biomarkers. Handb Exp Pharmacol 2009;192:29-60.

37. Freiberg MS, Cabral HJ, Heeren TC, Vasan RS, Curtis Ellison R; Third National Health and Nutrition Examination Survey. Alcohol consumption and the prevalence of the metabolic syndrome in the US: a cross-sectional analysis of data from the Third National Health and Nutrition Examination Survey. Diabetes Care 2004;27:2954-9.

38. Kim SK, Hong SH, Chung JH, Cho KB. Association between alcohol consumption and metabolic syndrome in a community-based cohort of Korean adults. Med Sci Monit 2017;23:2104-10.

39. Korean Society of Cardiometabolic Syndrome. Metabolic syndrome fact sheet in Korea 2018. Incheon: Korean Society of Cardiometabolic Syndrome; 2018.

40. Aurrekoetxea JJ, Murcia M, Rebagliato M, Lopez MJ, Castilla AM, Santa-Marina L, et al. Determinants of self-reported smoking and misclassification during pregnancy, and analysis of optimal cut-off points for urinary cotinine: a cross-sectional study. BMJ Open 2013;3:e002034.

41. Ali AT. Polycystic ovary syndrome and metabolic syndrome. Ceska Gynekol 2015;80:279-89.

42. Pasquali R. Metabolic syndrome in polycystic ovary syndrome. Front Horm Res 2018;49:114-30. 